\title{
Modelación de los cambios químicos en suelos inducidos por la forestación de pastizales naturales en ecosistemas de llanura
}

\author{
Claudio R. Mujica ${ }^{1, \bigotimes}$; Germán M. Milione'; Sergio A. Bea \& Esteban G. JobbáGy² \\ ${ }^{1}$ Instituto de Hidrología de Llanuras, Universidad Nacional del Centro de la Provincial de Buenos Aires y CONICET. ${ }^{2}$ Grupo \\ de Estudios Ambientales, IMASL, Universidad Nacional de San Luis y CONICET, San Luis, Argentina.
}

\begin{abstract}
RESUMEN. El reemplazo de pastizales por forestaciones es uno de los cambios más extremos en el uso del suelo por su impacto en el suelo y en el funcionamiento de la vegetación. En los ecosistemas de llanuras, este cambio generó, en muchos casos, alteraciones químicas en los suelos como salinización, sodificación y alcalinización/ acidificación. Si bien los procesos hídricos, químicos y biológicos que tienen lugar fueron extensamente caracterizados, debido al acoplamiento entre estos procesos y su no-linealidad, la tarea de cuantificarlos es difícil y, hasta el momento, no existen estudios que los hayan evaluado. El presente trabajo intenta caracterizar, mediante la modelación del transporte reactivo, los procesos físico-químicos que tienen lugar en un pastizal forestado con Eucalyptus camaldulensis en la Pampa Deprimida (Castelli, provincia de Buenos Aires). Para ello, se utilizó información hidrológica y química (agua, suelo y plantas). El modelo numérico simula los flujos de agua y las potenciales reacciones químicas en el contexto de reproducir 1) las alteraciones hidrológicas que causa la forestación y por 2) la intrusión de agua salina profunda, 3) la absorción y la exudación de nutrientes por las raíces, y 4) la respiración y el reciclaje de solutos. Los resultados de la modelación sugieren que factores como la composición química de la napa freática, el intercambio catiónico de los materiales del suelo con la solución del suelo, la precipitación/disolución de minerales y la interacción con la rizósfera (toma/exclusión/ exudación de solutos) son los mecanismos dominantes que controlarían la dirección del cambio químico en el suelo (acidificación vs. alcalinización). Este cambio muestra contrastes marcados en distancias cortas ( $250 \mathrm{~m}$ ) desde el pastizal, pasando por el margen, hasta el centro del sector forestado. Finalmente, la intensidad y la dirección de los cambios, y la transformación del suelo dependerá tanto del nuevo ecosistema que los humanos imponen como de la naturaleza química e hidrológica del sitio.
\end{abstract}

[Palabras clave: cambio en el uso del suelo, salinización, sodificación, acidificación, alcalinización, modelo de transporte reactivo]

\begin{abstract}
Aвstract. Modeling soil chemical changes induced by afforestation of natural grasslands in large plain ecosystems. The replacement of grasslands by forests is one of the most extreme changes in land use due to its impact on soil and vegetation functioning. On large plain ecosystems, this shift causes, in many cases, soil chemical changes such as salinization, sodification and alkalization/acidification. Although the hydrological, chemical and biological processes involved have been extensively characterized, due to their coupling and non-linearity, their quantification is a hard task, and until the present day, they were not addressed by any research work. The present work intends to capture though reactive transport modeling, the physical-chemical processes that take place in an afforested grassland with Eucalyptus camaldulensis located on the Flooding Pampas (Castelli, province of Buenos Aires), using hydrological and chemical information (water, soil and plants). The numerical model simulates the water flows and chemical reactions in the context of reproduce 1) the hydrological changes induced by forest, and 2) the intrusion of deep saline water, 3) the absorption and exudation of nutrients by the roots, and 4) the respiration and solutes recycling. Modeling results suggest that factors such as the water table chemical composition, the cation exchange processes with the soil solution, the mineral precipitation/dissolution, and the interaction of the solution with the rhizosphere (solutes uptake/ exclusion/exudation), are the dominant mechanisms that control the direction of chemical change in the soil. This change shows marked contrasts in the short distance $(250 \mathrm{~m})$ from grassland passes through the outer belt and reaches the core of the afforested plot. Finally, the change intensity and direction, and transformation of soil will depend both on the new ecosystem that humans impose and on the chemical and hydrological nature of the site.
\end{abstract}

[Keywords: land use change, salinization, sodification, acidification, alkalization, reactive transport model]

Editor asociado: Gervasio Piñeiro
Recibido: 14 de Marzo de 2019

Aceptado: 12 de Agosto de 2019 


\section{INTRODUCCIÓN}

La intensificación en las actividades agropecuarias surgió como respuesta de la gran demanda de alimentos y productos primarios en el mundo (Ickowitz et al. 2019; Lal 2003). Esta tendencia condujo a una alta presión sobre la productividad del suelo, con el consecuente cambio en su uso para adaptarlo a diferentes actividades con rentabilidad económica (e.g., agricultura, silvicultura, etc.). La transición entre pastizales y forestaciones fue uno de los cambios en el uso del suelo que impactó en su productividad (Fuchs et al. 2015; Ramankutty et al. 2018). La forestación de pastizales impacta localmente tanto en el balance de agua del sistema como en el balance de solutos y en las propiedades físico-químicas del suelo (Hong et al. 2018; Nosetto et al. 2007). Existen muchos trabajos que describen las causas y los efectos derivados de estos cambios en los usos del suelo (Heuperman 1999; Jobbágy and Jackson 2007; Nosetto et al. 2008), pero los procesos implicados no fueron suficientemente estudiados desde el punto de vista cuantitativo, ni de manera comprensiva y simultánea (Hong et al. 2018).

Los árboles presentan diferencias morfológicas y fisiológicas distintivas con las especies herbáceas, entre ellas un mayor LAI (índice de área foliar), un sistema radical más profundo, menor albedo, mayor rugosidad aerodinámica e intercepción de las precipitaciones. Estas diferencias se traducen en una mayor capacidad para usar el agua del sistema (Calder 1998; Engel et al. 2005; Jackson et al. 2001; Nosetto et al. 2012). El mayor uso del agua por parte de las forestaciones generalmente se acompañada por una redistribución fuerte de los solutos presentes en el suelo, y por una modificación de las propiedades físico-químicas de éste (e.g., alcalinización, sodificación, etc.). El carbono y nutrientes como el calcio $\left(\mathrm{Ca}^{2+}\right)$, el magnesio $\left(\mathrm{Mg}^{2+}\right)$ y el nitrógeno $(\mathrm{N})$ del suelo disminuyen, mientras que otros como el sodio $\left(\mathrm{Na}^{+}\right)$y el cloro $\left(\mathrm{Cl}^{-}\right)$se concentran, lo cual afecta su fertilidad (Berthrong et al. 2009; Hong et al. 2018). Cabe destacar que la magnitud de estos cambios podría modificar el funcionamiento bioquímico y "estructural" del suelo, y generar, por ejemplo, acidificación, que reduce su actividad microbiana (Kunito et al. 2016) e incrementa la pérdida de nutrientes por lixiviación (Bowman et al. 2008). Por otro lado, la acumulación residual (luego de la salinización) de $\mathrm{Na}^{+}$causa: 1) la dispersión de coloides, lo que disminuye la estabilidad estructural del suelo y su porosidad, con la consecuente disminución en la capacidad de conducción y almacenamiento de agua y gases, y 2) un aumento de la movilidad y la pérdida de materia orgánica, lo que compromete de forma irreversible su fertilidad (van Breemen and Buurman 2002; Hong et al. 2018; Jobbágy et al. 2017; Sumner 1993).

Conocer y cuantificar los procesos que afectan la productividad de los suelos en función de su uso es crucial para una óptima gestión hídrica de las cuencas asociadas, en donde se desarrollan estas actividades (Nosetto et al. 2007). Varios trabajos analizaron cualitativamente los potenciales mecanismos que controlan la acidificación y la salinización de los suelos en pastizales forestados (Dakora and Phillips 2002; Hong et al. 2018; Jobbágy and Jackson 2004). Entre estos mecanismos propuestos podemos citar: a) la intrusión de agua profunda producto de la descarga inducida por la forestación, b) la toma selectiva de nutrientes, c) la exudación de solutos por parte de las raíces, d) el reciclaje de solutos del sistema, y e) la mayor tasa de respiración en el suelo forestal.

El ingreso de agua profunda al sistema (a) es consecuencia de la descarga hídrica local generada por el gran consumo de agua por parte de la forestación. Antes de que se establezca la forestación, el nivel freático se caracteriza químicamente por agua proveniente de recarga de lluvia local; sin embargo, luego del cambio de vegetación, esta agua residente se consume rápidamente y es reemplazada por otra, potencialmente de una fuente más profunda, y con diferente composición química (Heuperman 1999; Jobbágy and Jackson 2007). La toma selectiva de nutrientes por parte de la rizósfera (b) genera acumulación o empobrecimiento de ciertos solutos en el suelo (Nye 1981; Ae et al. 2001; Hong et al. 2018). Por otra parte, los exudados de la raíz (c) consisten en una mezcla compleja de aniones de ácidos orgánicos, fitosideróforos, azúcares, vitaminas, aminoácidos, purinas, nucleósidos, iones inorgánicos (e.g., $\mathrm{HCO}_{3}^{-}, \mathrm{OH}^{-}, \mathrm{H}^{+}$), moléculas gaseosas $\left(\mathrm{CO}_{2}, \mathrm{H}_{2}\right)$ y enzimas. En general, los árboles consumen más cationes que aniones y liberan $\mathrm{H}^{+}$para mantener el balance de cargas eléctricas en la solución acuosa (Dakora and Phillips 2002).

Con respecto al reciclado de nutrientes (d), Jobbágy y Jackson $(2001,2003)$ resaltan que este mecanismo sería uno de los dominantes 
en la acidificación y la salinización de los suelos forestados. Este último causaría el enriquecimiento superficial de solutos, incluyendo aquellos que se encuentran incluso como minoritarios en las aguas subterráneas naturales. Esto ocurriría porque los solutos se acumulan en la biomasa de la forestación, y después se depositan sobre la superficie por medio de la hojarasca, desrame, desprendimiento de corteza, etc. La mayor tasa de respiración en los suelos forestados (e) puede causar acidificación (Epron et al. 2004; O’Connel 1987). Esta respiración más elevada estaría dada por el mayor volumen de raíces y por un aumento en la actividad microbiológica del suelo debido a la mayor cantidad de residuos. Además, la inyección de $\mathrm{CO}_{2}$ altera el equilibrio químico de la calcita y otros carbonatos, y puede generar su disolución (Appelo and Postma 2004). Sin embargo, existen varios autores que indican un comportamiento contrario, con una menor tasa respiratoria en suelos forestales (Saviozzi et al. 2001).

Para evaluar o predecir el impacto de la actividad forestal en estos ambientes es necesario tener en cuenta un gran número de procesos físicos, químicos y biológicos que actúan acoplados. Si se tiene en cuenta la complejidad y la no-linealidad de la interacción de estos procesos, es necesario usar herramientas que permitan capturar y validar estos mecanismos, tales como los modelos de transporte reactivo (van Breemen et al. 1983; Steefel et al. 2005). En la actualidad se desarrollaron varios modelos de este tipo que incluyen los procesos acoplados mencionados anteriormente (Bea et al. 2012; Mayer et al. 2012; Steefel et al. 2014). Estos tienen en cuenta: 1) el flujo del agua y el transporte de solutos a través de la matriz del suelo, 2) procesos químicos como, por ejemplo, la formación de complejos acuosos, el intercambio catiónico, la disolución y precipitación de minerales, reacciones acido/ base, etc., y 3) procesos de la rizósfera tales como toma de agua y nutrientes, exudación y respiración (Gerard et al. 2017; Hopmans and Bristow 2002; Hinsinger et al. 2005; Nowack et al. 2006). Sin embargo, a pesar de su relevancia, hasta el presente no se utilizaron modelos para estudiar los procesos asociados a la rizósfera de forestaciones freatófitas (e.g., eucaliptos) acoplados a la química del suelo.

El objetivo del presente trabajo fue identificar y validar aquellos mecanismos y condiciones que conducen a los cambios químicos en el suelo en pastizales forestados, haciendo uso del transporte reactivo. El modelo utilizado fue MIN3P (Mayer et al. 2002, 2012; Bea et al. 2012, 2013), que fue modificado para tener en cuenta los fuertes desbalances eléctricos que tienen lugar en la rizósfera de las forestaciones. Este modelo se aplicó a una típica parcela de pastizal forestada en la llanura pampeana con evidencias de salinización de suelo (Jobbágy and Jackson 2004, 2007; Engel et al. 2005).

\section{Materiales y Métodos}

\section{Descripción de la zona de estudio}

La zona de estudio se encuentra en la Pampa Deprimida. Se caracteriza por bajas pendientes topográficas y por flujos de agua preferencialmente verticales (Varni and Usunoff 1999). Esta amplia región de alrededor de 8 millones de hectáreas está cubierta por depósitos de loess del PleistocenoHoloceno tardío que forman un manto de espesor variable. El tipo de suelo más común en este ambiente de llanura son de tipo salinosódico (Natracuoles y Natracualfes, US Soil Taxonomy; Solonetzes, según FAO Soil Map), desarrollados a partir de estos sedimentos, adecuados para la ganadería extensiva en pastoreo (Teruggi 1957; Zárate 2003; Viglizzo et al. 2001). El sitio de estudio, sin embargo, se encuentra en una formación eólica típica de la zona con una posición elevada respecto del terreno circundante, con aptitud para la agricultura y la ganadería. El suelo es un Hapludol (del tipo de la serie "Los Naranjos") (INTA 2011) que cubre un suelo erosionado, más antiguo (horizontes II). La secuencia de horizontes en el sitio es: A $(0-15 \mathrm{~cm}), \mathrm{AC}(15-$ $35 \mathrm{~cm})$, IIB $(35-75 \mathrm{~cm})$ y IIC $(75-300 \mathrm{~cm})$. Sus bajas pendientes topográficas $(<0.1 \%)$ y la erosión eólica del Cuaternario crearon una red de drenaje pobre. Esto, sumado al clima subhúmedo a húmedo (800-1000 mm/año), resulta en un acuífero somero en toda la región (Tricart 1973), y con un tipo de agua dominante del tipo $\mathrm{Na}-\mathrm{HCO}_{3}$ (Zabala et al. 2016; Carretero et al. 2013). Estas aguas subterráneas incrementan su contenido de sales desde las zonas de recarga hacia los puntos de descarga, y se observan procesos de salinización y alcalinización aguas abajo, con la consecuente pérdida de fertilidad de suelos potencialmente productivos (Jobbágy et al. 2017).

La zona de estudio del presente trabajo involucra una transecta que recorre pastizal/ forestación ubicada en la localidad de Castelli 
$\left(-36^{\circ} 02.0^{\prime}-57^{\circ} 50.3^{\prime}\right)$ (Figura 1). Este sitio fue forestado con Eucalyptus camaldulensis en 1951, en una matriz de pastizal típico de la Pampa Deprimida (Cabrera 1976; Burkart et al. 2005). El manejo al cual fueron sometidos es el típico para la zona; el pastizal fue pastoreado, la forestación no tuvo ninguna intervención y no se realizaron prácticas de riego ni de fertilización. Más detalles sobre el sitio de estudio se pueden ver en Jobbágy y Jackson (2004) y en Engel et al. (2005).

La precipitación media en el sitio de estudio promedia $\sim 980 \mathrm{~mm} / a$ ño en 53 años y los niveles freáticos varían entre $\sim 0.45$ y $\sim 5.5 \mathrm{~m}$ de profundidad (ese segundo valor coincide con la máxima profundidad observada que alcanzaron las raíces) (Jobbágy and Jackson 2004). Cabe destacar que en este sitio la forestación genera un descenso del nivel freático de $\sim 30$ a $70 \mathrm{~cm}$ respecto del pastizal. La tasa de traspiración de la forestación es de $\sim 2.16 \mathrm{~mm} /$ día, sostenida por un consumo de agua freática de $\sim 290$ a 390 mm/año (Engel et al. 2005). Este consumo genera un esquema de flujos (Figura 1b) laterales (entre 160 y 900 $\mathrm{mm} / \mathrm{año}$ ) que van desde la parcela herbácea hacia la forestación, así como también flujos verticales que provienen de aguas profundas (Engel et al. 2005; Jobbágy and Jackson 2004, 2007). El pH de suelo varía a lo largo de la transecta (Figura 1), con $\sim 8$ en el pastizal, $\sim 9$ en el margen exterior y $\sim 6.5$ en el centro de la forestación (Figura 2). De la misma manera, la química del suelo y del freático varía desde el pastizal hacia la forestación. El agua bajo la forestación es $\sim 5$ veces más salina, con un mayor contenido en $\mathrm{Cl}^{-}$y $\mathrm{Na}^{+}$(Tabla1). En el

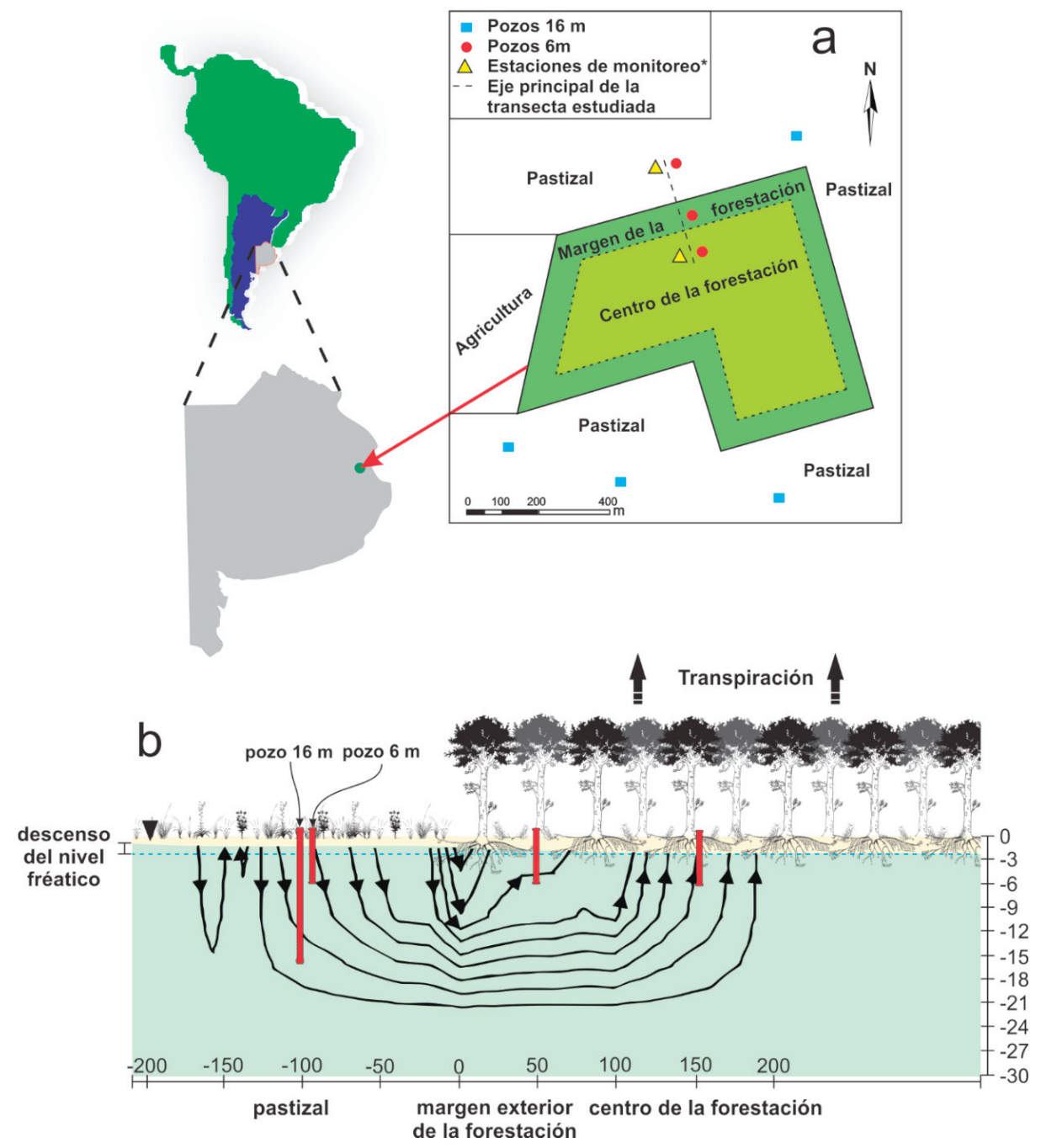

Figura 1. Ubicación y características de la transecta estudiada. *Estaciones donde se monitoreó el nivel freático y la traspiración. Las líneas de flujo en (b) fueron extraídas de Jobbágy y Jackson 2007.

Figure 1. Location and characteristics of the studied transect. *Stations where the water table and transpiration were monitored. The flow lines in (b) were extracted from Jobbágy and Jackson 2007. 
Tabla 1. Composición química de las aguas medidas y calculadas.

Table 1. Chemical composition of the measured and calculated waters.

\begin{tabular}{|c|c|c|c|c|c|c|c|c|c|c|c|c|c|}
\hline Muestras & $\begin{array}{l}\text { Posi- } \\
\text { ción } \\
(\mathrm{m})\end{array}$ & $\begin{array}{c}\text { EC } \\
(\mathrm{dS} / \mathrm{m})\end{array}$ & $\mathrm{pH}$ & $\begin{array}{l}\mathrm{Ca}^{+2} \\
\mathrm{mg} / \mathrm{L}\end{array}$ & $\mathrm{Mg}^{+2}$ & $\mathrm{Na}^{+}$ & & $\mathrm{Cl}^{-}$ & $\mathrm{SO}_{4}^{2+}$ & $\mathrm{CO}_{3}^{2+}$ & $\mathrm{HCO}_{3}^{+}$ & $\mathrm{Na}: \mathrm{Cl}$ & $\begin{array}{c}\mathrm{RSC}^{\mathrm{c}} \\
\mathrm{mmol} / \mathrm{L}\end{array}$ \\
\hline Colectores de lluvia & 100 & 0.00 & 5.91 & 0.71 & 0.18 & 0.53 & 0.59 & 0.71 & 0.10 & 0 & 0 & 1.15 & - \\
\hline Pastizal (6 m) & 100 & 0.87 & 7.73 & 58 & 28 & 46 & 38 & 15 & 11 & 12 & 537 & 4.73 & 6.4 \\
\hline Pastizal (16 m) & 100 & 1.24 & 7.77 & 40 & 26 & 161 & 34 & 70 & 36 & 14 & 725 & 3.55 & 10.0 \\
\hline Margen de forestación (6 m) & -50 & 2.02 & 7.96 & 31 & 36 & 250 & 42 & 264 & 104 & 76 & 752 & 1.75 & 11.3 \\
\hline Centro de forestación (6 m) & -150 & 4.21 & 7.26 & 186 & 112 & 478 & 23 & 1165 & 216 & 0 & 387 & 0.63 & -2.9 \\
\hline Mezcla MFP b & -50 & $1.74^{\mathrm{a}}$ & 7.69 & 72 & 42 & 239 & 40 & 238 & 71 & 0 & 540 & 1.55 & 5.35 \\
\hline Mezcla CFP b & -150 & $9.76^{\mathrm{a}}$ & 7.61 & 219 & 185 & 1646 & 85 & 2315 & 658 & 0 & 719 & 1.10 & -1.3 \\
\hline Agua de origen marino $^{\mathrm{d}}$ & - & 26.5 & 7.01 & 719 & 678 & 6630 & 118 & 10700 & 2900 & 0 & 659 & 0.96 & -35.0 \\
\hline
\end{tabular}

${ }^{a}$ calculadas según Miller et al. 1988. ${ }^{b}$ Calculadas por modelación inversa con PHREEQC para asemejarse a las proporciones encontradas en margen y centro de la forestación. ' $R S C$ es calculado como RSC $=\left(\left[\mathrm{HCO}_{3}^{+}\right]+\left[\mathrm{CO}_{3}{ }^{2+}\right]\right)$ $\left.\left(\left[\mathrm{Ca}^{+2}\right]\right)+\left[\mathrm{Mg}^{+2}\right]\right) \cdot{ }^{d}$ Extraído de BDH 2019.

a

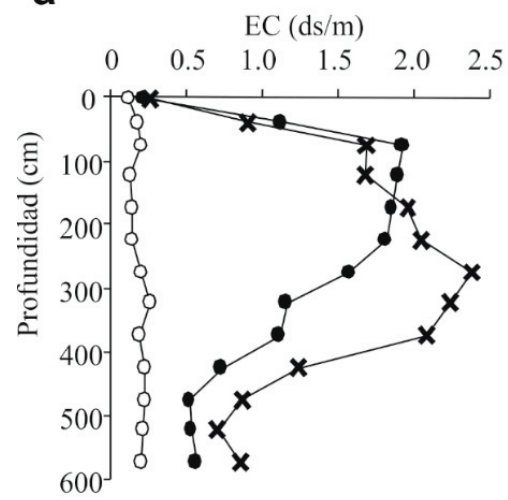

c

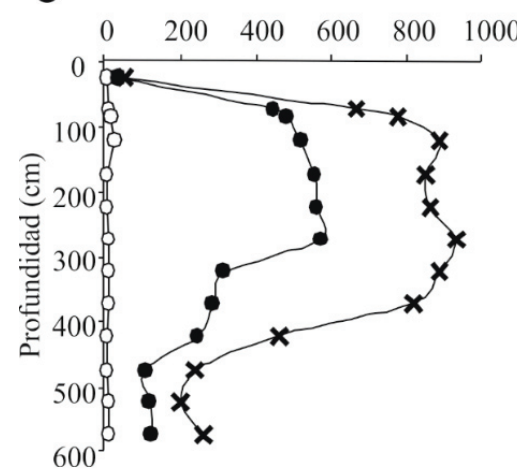

b

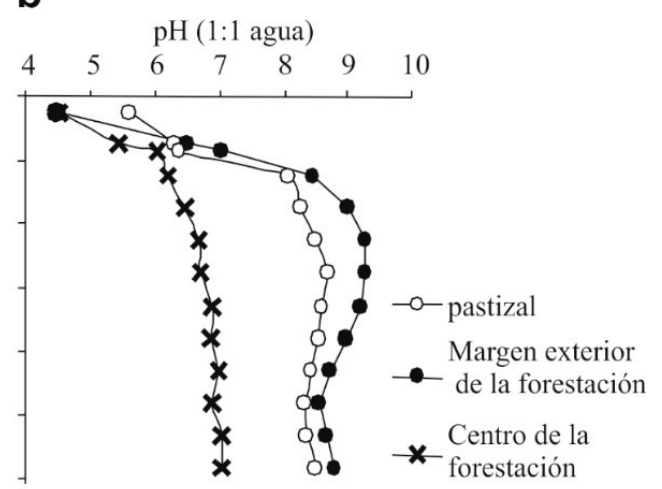

Porcentaje de $\mathrm{Na}$

d intercambiable (PSI, \%)

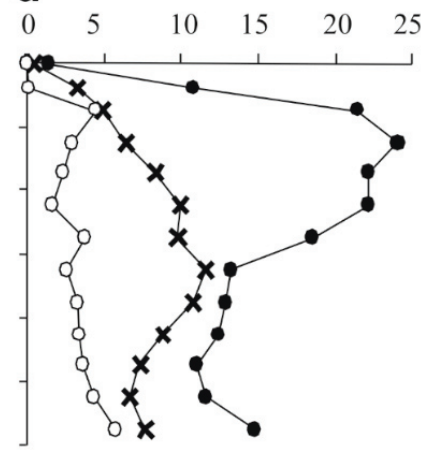

Figura 2. Características químicas de la transecta estudiada, extraído de Jobbágy y Jackson (2007). (a) La conductividad eléctrica (EC) corresponde con mediciones sobre una relación suelo:agua de 1:5; (b y c) Para pH y cloruro se usó una relación 1:1. (d) El porcentaje de sodio intercambiable (PSI) indica el porcentaje del complejo de cambio que está saturado por ese elemento. La posición de pastizal se hizo a $100 \mathrm{~m}$ de la forestación, mientras que margen y centro se ubicaron a 50 y $150 \mathrm{~m}$ hacia el interior de la misma, respectivamente.

Figure 2. Chemical characteristics of the studied transect, extracted from Jobbágy and Jackson (2007). (a) The electrical conductivity (EC) corresponds with measurements on a soil:water ratio of 1:5; (b and c) For $\mathrm{pH}$ and chloride, a 1:1 ratio was used. (d) The exchangeable sodium percentage (PSI) indicates the proportion of the exchange complex that is saturated with that element. The grassland position is $100 \mathrm{~m}$ away from the plantation, meanwhile outer belt and core positions are located 50 and $150 \mathrm{~m}$, respectively, towards the plantation interior. 


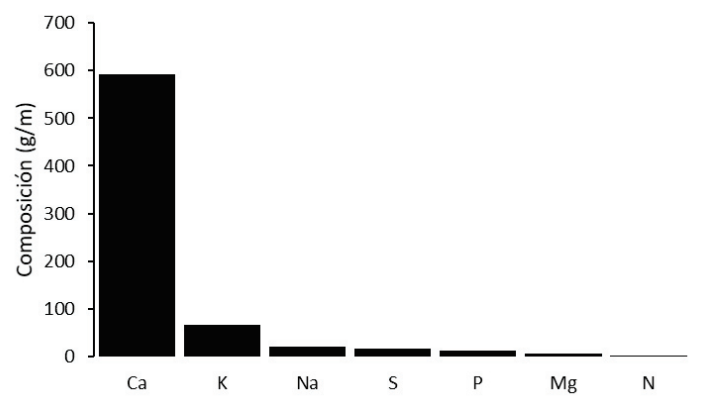

Figura 3. Composición de la biomasa de Eucalyptus camaldulensis. Para detalles metodológicos sobre la obtención de estos datos ver Jobbágy y Jackson (2003).

Figure 3. Biomass composition of Eucalyptus camaldulensis. For methodological details on obtaining this data see Jobbágy and Jackson (2003).

suelo hay, en promedio, $\sim 20 \%$ más de $\mathrm{Na}$ intercambiable, $\sim 800 \mathrm{~g} / \mathrm{m}^{2}$ más de $\mathrm{Cl}$ y entre $\sim 10$ y 30 veces más salinidad (Figura 2). La gran biomasa acumulada en la parcela forestal $(\sim 754 \mathrm{Mg} / \mathrm{ha})$ tiene una composición química que difiere en sus proporciones respecto del suelo y agua subterránea, lo que refleja el efecto de la toma selectiva de nutrientes (Figura 3).

\section{Dominio y características del modelo}

En base a los estudios mencionados más arriba (ver Engel et al. 2005; Jobbágy and
Jackson 2004, 2007) (Figura 1) se montó una serie de modelos de transporte reactivo con dominio unidimensional y un tiempo de 50 años. Para ejecutar estos modelos se usó el código MIN3P (Bea et al. 2012, 2016; Mater et al. 2002, 2012) y se le incorporó la funcionalidad de balancear eléctricamente el medio químico sometido a la toma diferencial de iones por las raíces (ver Anexo, Ecuación 8). El dominio del modelo consistió en un bloque de $30 \mathrm{~m}$ de profundidad de loess y un área unitaria $\left(1 \mathrm{~m}^{2}\right)$. El mismo se discretizó en 19 capas, cada una con propiedades físico-químicas diferentes (Figura 4 y Tabla A1 Anexo). Se partió de las condiciones químicas del suelo y agua iniciales medidas en la parcela de pastizal. La información de las propiedades físicas y químicas del suelo fueron recopiladas en trabajos previos cuyos detalles metodológicos pueden obtenerse en Jobbágy y Jackson (2003, 2004, 2007) y Engel et al. (2005).

En la parte superior del dominio (Figura 4) se impuso un caudal que representa el volumen promedio de precipitaciones ( $\mathrm{mm} /$ día), con su respectiva composición química recolectada bajo la superficie del suelo de la forestación; así se considera el aporte de nutrientes/ solutos reciclados (ver Jobbágy and Jackson 2003, 2007). En la parte inferior se impone el nivel freático variable en el tiempo (basado
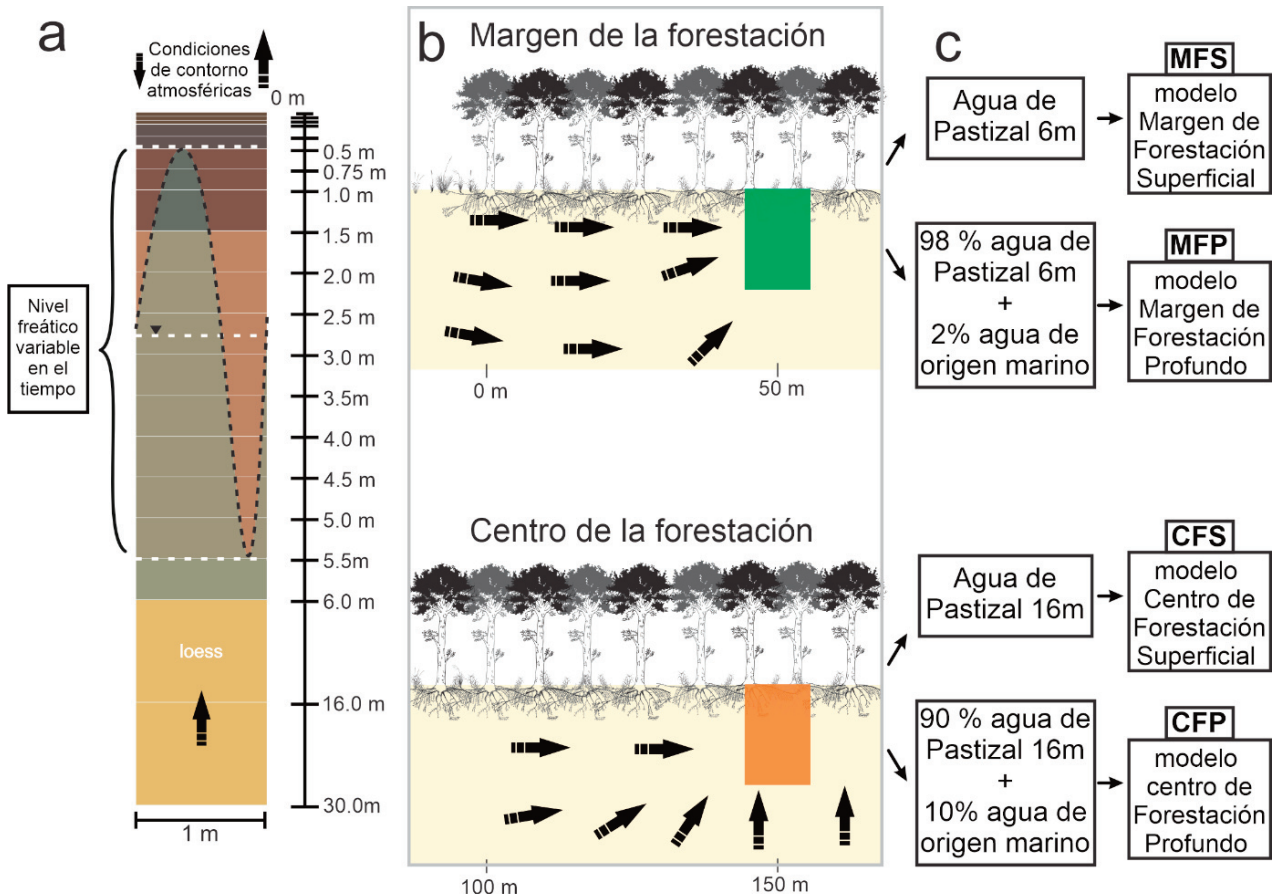

Figura 4. (a) Dominio del modelo, (b) modelo conceptual de flujo basado en Jobbágy y Jackson (2007), y (c) modelos implementados.

Figure 4. (a) Model domain, (b) conceptual flow model based on Jobbágy and Jackson (2007) and (c) models implemented. 
sobre mediciones directas y observación de indicadores edáficos) (Jobbágy and Jackson 2004; Engel et al. 2005). Con respecto a la tasa de consumo de agua por parte de las raíces se usó el modelo y parámetros propuestos por Battaglia y Sands (1997), en el que la toma de agua del suelo está restringida por el agua disponible en el perfil y por evapotranspiración del cultivo en condiciones estándar. Ésta se calculó según Allen et al. (2006) con los coeficientes de cultivo para Eucalyptus camaldulensis tomados de Besteiro (2014). Se usó la serie de transpiración medida en el sitio de estudio por Engel et al. (2005) como punto de comparación para validar el modelo. Las raíces se distribuyeron en los primeros $6 \mathrm{~m}$ del dominio en base a mediciones realizadas en el primer metro y se extrapoló entre 1 y 6 $\mathrm{m}$, profundidad en base a la observación (no medición) de la presencia de raíces hasta esa profundidad, usando una función de densidad decreciente (Jackson et al. 1996).

La toma de nutrientes por parte de la rizósfera fue calibrada de acuerdo con la cantidad de solutos acumulada en la biomasa para el caso de $\mathrm{Na}^{+}, \mathrm{Ca}^{2+}, \mathrm{K}^{+}, \mathrm{Mg}^{2+}, \mathrm{N}, \mathrm{S}$ y $\mathrm{P}$ (Figura 3). Por otro lado, para el caso de $\mathrm{Cl}^{-}$se asumió que es excluida completamente por las raíces (Teakle and Tyerman 2010), mientras que $\mathrm{H}^{+}$y $\mathrm{CO}_{3}^{-2}$ entran en las raíces por la vía pasiva (Amiro and Ewing 1992). Para simular la respiración de las raíces sumada a la de la microbiota del suelo se consideró una tasa de liberación constante de $\mathrm{CO}_{2}$ de acuerdo con mediciones realizadas en situaciones similares (Epron et al. 2004; O'Connel 1987; Raich and Tufekcioglu 2000). Finalmente, para compensar eléctricamente el agua circundante a las raíces se utilizó consumo/producción de $\mathrm{H}^{+}$(ver Ecuación 8 en Anexo). Además, este modelo tiene en cuenta el intercambio catiónico con el suelo, la precipitación y la disolución de calcita, el intercambio gaseoso con la atmósfera y la formación de complejos acuosos (ver Anexo para más detalles sobre el modelo).

De acuerdo con el modelo de patrón de flujo propuesto por Jobbágy y Jackson (2007) (Figura 1b) se plantearon dos escenarios principales; uno, en el que 1) el agua que ingresa al modelo por el contorno inferior tiene la química del agua medida en el pastizal a $6 \mathrm{~m}$ de profundidad, y otro en el que 2) se considera la química del mismo sitio, pero a $16 \mathrm{~m}$ de profundidad. Dichos escenarios intentan reproducir, como una primera aproximación, respectivamente, los procesos hidrogeoquímicos observados tanto en el margen exterior como en el centro de la forestación (Figura 4). Llamaremos a estos dos modelos Margen de Forestación (MFS) y Centro de la Forestación (CFS), siglas en las que la $S$ final se refiere a que las aguas que ingresan al sistema son de acuíferos superficiales observados in situ (freatímetros de 6 y 16 m) (Tabla 1). Además, se plantearon otros dos modelos alternativos que intentan evaluar la intrusión de agua profunda. En estos últimos casos, el agua que ingresa desde el contorno inferior corresponde a una mezcla de agua residente en el acuífero con otra más salina y profunda que descargaría en la forestación (potencialmente identificada aquí como descarga de agua de origen marino) (Carol et al. 2008). Las proporciones de estas mezclas ( $2 \%$ y $10 \%$ en el modelo del margen y el centro de la forestación, respectivamente) fueron determinadas con el modelo PHREEQC (Parkhurst and Appelo 1999), partiendo de las aguas de 6 y $16 \mathrm{~m}$ encontradas en el pastizal, mezclándolas con agua de origen marino (BDH 2019) para que se aproximen a las proporciones químicas de las aguas encontradas en margen y centro de la forestación a $6 \mathrm{~m}$, respectivamente (Tabla 1 , Figura 4). Este segundo grupo de escenarios se identificarán siguiendo la misma lógica que los anteriores como MFP y CFP, siglas en las que la letra $P$ hace referencia a que el agua que ingresa al sistema tiene un aporte de origen profundo (Tabla1).

Los resultados del modelo se compararán con las mediciones realizadas en el sito (Figura 2). Para comparar de forma correcta el aumento en la salinidad en el margen y en el centro de la forestación se normalizaron las mediciones de conductividad eléctrica $(\mathrm{EC}, \mathrm{dS} / \mathrm{m}$ ) respecto del valor inicial que corresponde con el medido en el pastizal (ECfinal/ECinicial). Los resultados del modelo (mol/L) se transformaron en EC (dS/m) usando la relación propuesta por Miller et al. (1988) y posteriormente se normalizaron de la misma manera que las mediciones.

\section{RESUltAdOS Y DISCUSIÓN}

En términos generales, los modelos reprodujeron de forma satisfactoria los cambios químicos observados en los suelos de parcelas forestadas. En el modelo MFS, la magnitud de la salinización fue menor ( 1.5 veces) (Figura 5a) que la observada y descrita 

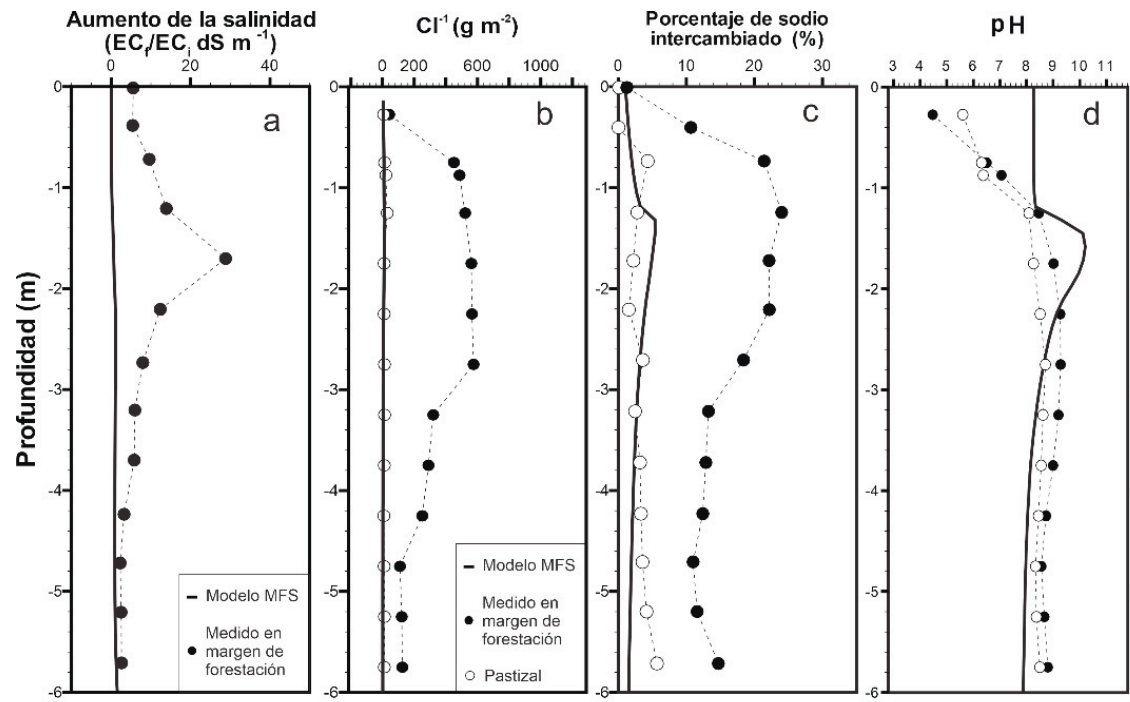

Calcita precipitada $(-)^{*}$
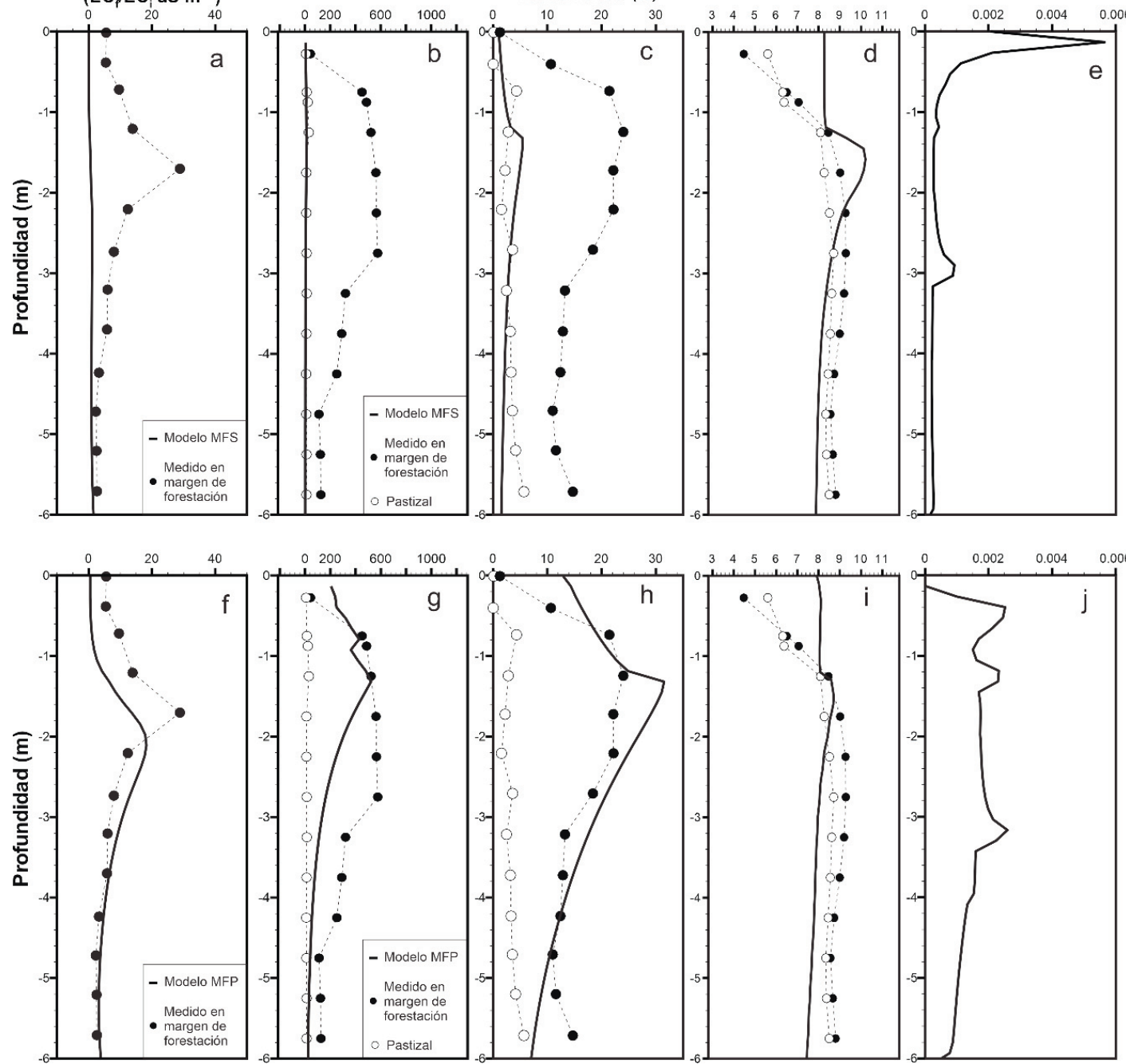

Figura 5. Resultados de campo y modelación para el margen de la forestación. En a, b, c y d, resultados del modelo Margen de Forestación Superficial (MFS) comparados con las observaciones en el pastizal y en el margen de la forestación. De la misma manera, en f, g, h, e i se comparan con los resultados del modelo Margen de Forestación Profundo (MFP). El aumento de la salinidad fue calculado como ECfinal/ECinicial (dS/m) para los datos medidos y modelados. *El volumen de calcita precipitada en MFS y MFP, respectivamente es calculado como volumen de mineral/volumen de suelo.

Figure 5. Field data and model results for forest outer belt. In a, b, c and d are shown the results of Margen de Forestación Superficial (MFS, translated as Shallow Forest Outer Belt) model, compared with grassland and outer belt field data. In the same way, in f, $g$, h, and i are compared the results of Margen de Forestación Profundo (MFP, translated as Deep Forest Outer Belt) model, with the same field data. The increase in salinity was calculated as ECfinal/Initial EC $(\mathrm{dS} / \mathrm{m})$ for the measured and modeled data. *Precipitated calcite volume was computed as: mineral volume/soil volume.

por Jobbágy et al. 2007 ( 20 veces) (Figura 2). Se debe tener en cuenta que comparar el aumento de la salinidad (EC final/EC inicial) medido con el simulado implica considerar que la EC se midió en una relación suelo (seco): agua destilada de 1:5 (Jobbágy and Jackson 2007) y que no es una medición directa del agua de poro del suelo. Por otro lado, el modelo de Miller et al. (1988) aplicado sobre los resultados simulados contiene información empírica y supuestos, y no considera la conductividad de las partículas del suelo (Miller et al. 1988) (ver Ecuación 11 en Anexos). Por estos motivos, aun normalizando la variable respecto del valor inicial (final/inicial), el aumento en la salinidad se comparará en términos aproximados. La acumulación de $\mathrm{Cl}^{-}$en este escenario fue de sólo $14 \mathrm{~g} / \mathrm{m}^{2}$, similar a los valores máximos observados en el pastizal no forestado, pero inferiores a los $600 \mathrm{~g} / \mathrm{m}^{2}$ observados en el margen de la forestación. Además, la acumulación de $\mathrm{Na}^{+}$ 
como complejo de cambio llega a valores de $\sim 5 \%$, mientras que los valores observados llegan a $\sim 25 \%$ (Figura 5 c y d). Con respecto a la distribución del $\mathrm{pH}$ en el suelo, este modelo reproduce una ligera alcalinización, alcanzando valores de $\mathrm{pH}$ máximos de 10 de (Figura 5d). Por otro lado, el modelo predice la formación de calcita en todo el perfil como resultado de la disponibilidad de $\mathrm{Ca}^{2+}$ desplazado del complejo de cambio por el aumento en el PSI (Figura 5e).

Con respecto al escenario MFP, la salinización ( 30 veces) (Figura 5f), la acumulación de $\mathrm{Cl}^{-}$ $\left(\sim 600 \mathrm{~g} / \mathrm{m}^{2}\right)$ (Figura 5g), el PSI ( 30\%) (Figura $5 \mathrm{~h})$ y el pH $(\sim 8)$ ( Figura 5i) son consistentes con las observaciones en ese sector. Estos resultados (MFS comparado con MFP) hacen evidente la existencia del ingreso de agua salina (profunda) para alcanzar las transformaciones químicas observadas. La mayor liberación de $\mathrm{Ca}^{2+}$ en este escenario (es decir, desplazado del complejo de cambio por el $\mathrm{Na}^{+}$), induce una mayor precipitación de carbonato de calcio (Figura $6 \mathrm{~h}$ ).

Para el caso del modelo CFS, la salinización predicha en este escenario es de hasta $\sim 40$ veces la observada en el pastizal (Figura 6a), la acumulación de $\mathrm{Cl}^{-}$en el suelo fue de hasta de $600 \mathrm{~g} / \mathrm{m}^{2}$ (Figura 6b), y a pesar de que este escenario reproduce un aumento del PSI, éste llega a valores de 9\%, mientras que los máximos observados en el centro de la forestación alcanzan $\sim 14 \%$ (Figura 6c). Con respecto a la distribución del $\mathrm{pH}$ del suelo, este escenario reproduce la acidificación observada en el centro de la forestación (pH 6) (Figura 6d). En el escenario CFP, el aumento de la salinidad se mantuvo en $\sim 40$ veces (Figura $6 \mathrm{e}$ ), el $\mathrm{Cl}^{-}$acumulado alcanzó $\sim 800 \mathrm{~g} / \mathrm{m}^{2}$ (Figura $6 f$ ), el PSI fue de $\sim 10 \%$ (Figura $6 \mathrm{~g}$ ) y el $\mathrm{pH}$
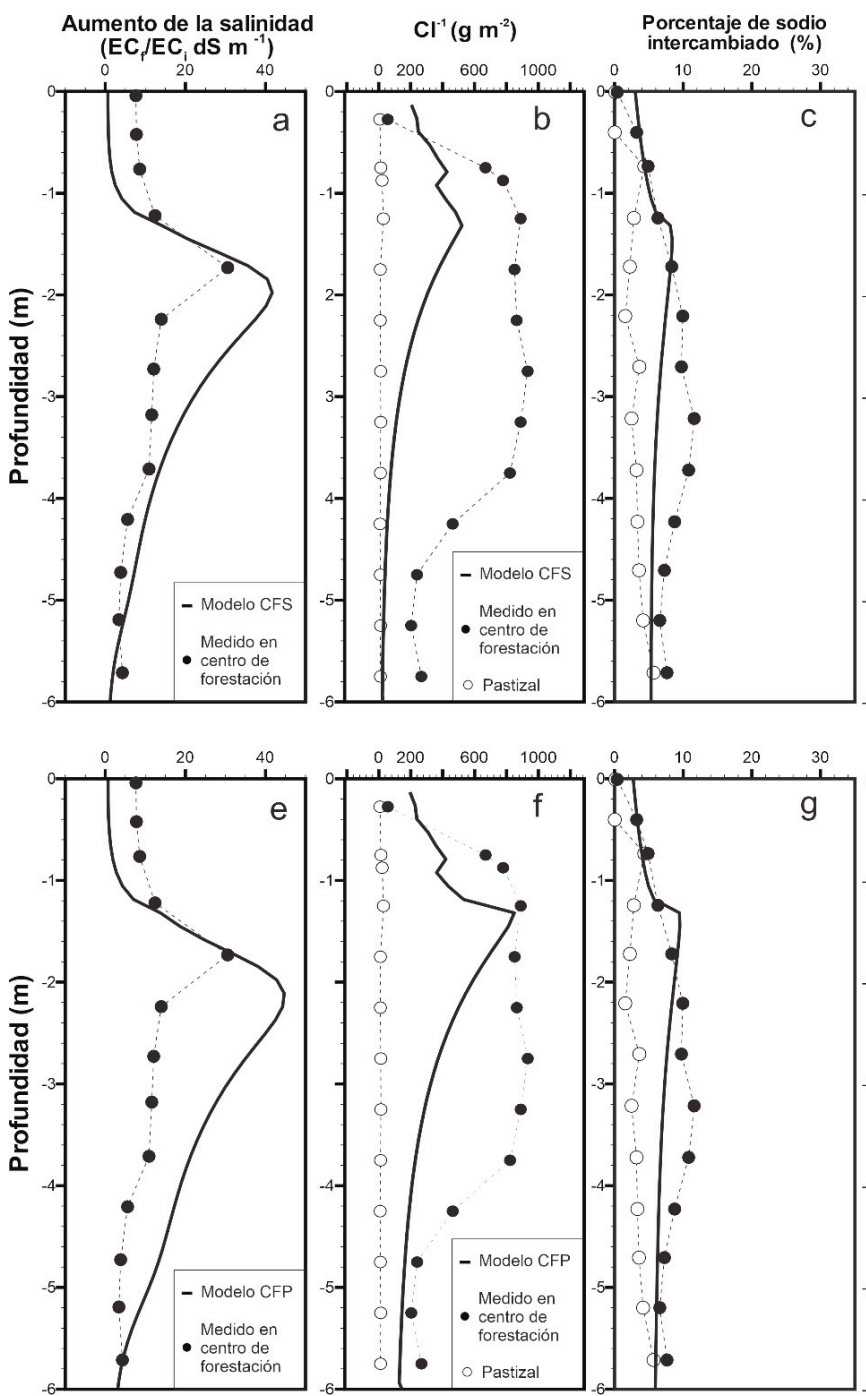

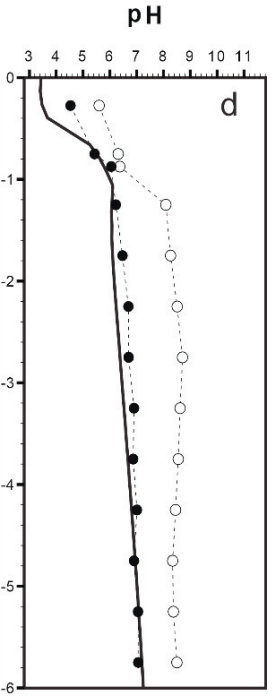

Figura 6. Resultados de campo y modelación para el centro de la forestación. En $a, b$, c y d, resultados del modelo Centro de Forestación Superficial (CFS) comparados con las observaciones en el pastizal y en el centro de la forestación. De la misma manera, en $\mathrm{f}, \mathrm{g}$, h e i se comparan con los resultados del modelo Centro de Forestación Profundo (CFP). El aumento de la salinidad fue calculado como ECfinal/ECinicial $(\mathrm{dS} / \mathrm{m})$ para los datos medidos y modelados.

Figure 6. Field data and model results for forest core. In $\mathrm{a}, \mathrm{b}, \mathrm{c}$ and $\mathrm{d}$, the results of the Centro de Forestación Superficial (CFS, translated as Shallow Forest Core") model are compared with grassland and forest core field data. In the same way, in $\mathrm{f}$, $\mathrm{g}, \mathrm{h}$, e $\mathrm{i}$ the results of Centro de Forestación Profundo (CFP, translated as Deep Forest Core") model are compared with the same field data. The increase in salinity was calculated as ECfinal/ Initial EC $(\mathrm{dS} / \mathrm{m})$ for the measured and modeled data. 
del suelo fue $\sim 6$ (Figura 6 h). En este conjunto de escenarios (CFS y CFP), el agua profunda aporta la concentración de cloruros necesaria para reproducir los valores medidos que de otra manera no se hubieran podido alcanzar. Cabe destacar que una menor relación molar $\mathrm{Na}$ Cl (Tabla 1) tiene impacto directo en el control del pHya que habría una compensación eléctrica por parte de la rizósfera por el exceso de aniones. Sin embargo, esta acidez generada por el desbalance eléctrico estaría controlada por la disolución de los carbonatos presentes previamente en el sistema (resultados no mostrados).

La precipitación de carbonatos tiene mayor ocurrencia en el margen de la forestación (MFS y MFP), ya que en estos escenarios existe una fuente de $\mathrm{CO}_{3}$ proveniente de la respiración y de la liberación de $\mathrm{Ca}^{2+}$ desde el complejo de cambio desplazado por la acumulación de $\mathrm{Na}$. En este contexto, ante un exceso de carbonatos, prácticamente todo el $\mathrm{Ca}^{2+}$ es precipitado y, dado el aumento de la concentración de la solución, se genera una alcalinización fuerte determinada por el equilibrio $\mathrm{CO}_{3}^{-2}+\mathrm{H}_{2} \mathrm{O}$ $\mathrm{HCO}_{3}{ }^{-}+\mathrm{OH}^{-}$(van Breemen and Buurman 2002). Una relación $\left(\mathrm{HCO}_{3}{ }^{-}+\mathrm{CO}_{3}^{-2}\right):\left(\mathrm{Ca}^{+2}+\mathrm{Mg}^{+2}\right)$, $\mathrm{O}$ carbonato de sodio residual (RSC), mayor a 1 es un indicador de que el sistema evolucionará en esta dirección (Jobbágy et al. 2017).

No obstante, al considerar estas relaciones es necesario tener en cuenta paralelamente los diferentes procesos involucrados. En primer lugar, la demanda de las raíces competiría por $\mathrm{Ca}^{2+}$ con la precipitación de calcita, lo que podría suponer entonces un efecto aditivo respecto de la alcalinización. Sin embargo, la toma de $\mathrm{Ca}^{2+}$ sería acompañada por la liberación de $\mathrm{H}^{+}$desde las raíces para compensar eléctricamente el agua del suelo en la rizósfera (van Breemen et al. 1983), generando el efecto contrario. En segundo lugar, es necesario tener en cuenta la relación $\mathrm{Na}^{+}: \mathrm{Cl}^{-}$del agua que es transpirada, ya que la mayor exclusión de $\mathrm{Cl}^{-}$comparado con $\mathrm{Na}^{+}$ (Figura 3), sumado al intercambio de $\mathrm{Na}^{+}$en el complejo de cambio puede generar un exceso de aniones. Así, en los escenarios CFS y CFP, la baja relación $\mathrm{Na}^{+}: \mathrm{Cl}^{-}$del agua sometida a la exclusión generara un exceso de aniones y un descenso del $\mathrm{pH}$ a causa de la liberación de $\mathrm{H}^{+}$(Tabla 1).

En estas condiciones, la calcita no precipita, por el contrario, la que previamente se encontraba en el suelo actuaría controlando la acidez, previniendo valores de $\mathrm{pH}$ más bajos entre 1 y $6 \mathrm{~m}$ de profundidad. En este sentido, cobra mayor importancia la relación $\mathrm{Na}^{+}: \mathrm{Cl}^{-}$, ya que, por ser las especies químicas más excluidas por las raíces, y por estar en proporciones relativamente altas en el agua freática, son las que más se acumulan en los suelos por causa de la forestación. En el margen de la forestación el agua que se está traspirando tiene una relación $\mathrm{Na}^{+}: \mathrm{Cl}^{-}$alta, por lo que el sistema se alcaliniza y hay una mayor saturación de $\mathrm{Na}^{+}$en el complejo de cambio.

\section{Conclusiones}

Los modelos numéricos de transporte reactivo aplicados aquí reproducen de forma satisfactoria la situación actual observada en el sitio de estudio. La hidroquímica de las aguas subterráneas sería responsable de la magnitud y la dirección (alcalinización/acidificación) de los cambios químicos. En este sentido, aquellos escenarios simulados que incluían mezcla con aguas de mayor salinidad presentan un mejor ajuste de los cambios químicos observados en estos suelos. Además, este hecho es consistente con los bajos contenidos de ${ }^{3} \mathrm{H}$ medidos en el centro de la forestación por Jobbágy y Jackson (2007), típicos de aguas subterráneas con altos tiempos de residencia en el acuífero.

Las interacciones entre relaciones iónicas de la solución del suelo, complejo de cambio, precipitación de minerales, toma/exclusión/ exudación de las raíces, imponen equilibrios difíciles de predecir por medio de indicadores aislados (e.g., $\mathrm{RSC}, \mathrm{Na}^{+}$:Cl', etc.); así, el sistema puede evolucionar en direcciones opuestas. Los modelos conceptuales presentados aquí fueron objeto de duda en muchos trabajos a lo largo de varias décadas (Hong et al. 2018, 2019; Jobbágy and Jackson 2001, 2003, 2004, 2007; Nilsson et al. 1982). A través de la modelación numérico del transporte reactivo se pudieron capturar los complejos procesos acoplados inducidos por el cambio en el uso del suelo entre pastizal y forestación. La salinización, acidificación o alcalinización de los suelos forestados sólo se dan con una combinación de condiciones específicas, y existirían situaciones en la que es posible la producción forestal sin mayores impactos, incluso en ecosistemas similares a los aquí estudiados, en las pampas y a lo largo del mundo (Jobbágy and Jackson 2004; Nosetto et al. 2009; Milione et al. 2018). El impacto ecológico de la forestación de pastizales es un proceso que debe ser evaluado tanto en la magnitud de los cambios que se imponen sobre el ecosistema vegetal, como en las características hidrogeoquímicas del sitio, ya que diferencias en este punto pueden 
resultar en alteraciones tan diferentes como las observadas en el margen y el centro de la forestación.
Agradecimientos. Este trabajo fue financiado por becas de investigación de FONCYTMINCyT (PICT-2015-0744), Argentina.

\section{REFERENCIAS}

Ae, N., J. Arihara, K. Okada, and A. Srinivasan. 2001. Plant nutrient acquisition: new perspectives. Springer Science and Business Media. Pp 71-100.

Allen, G.R., L. S. Pereira, D. Raes, and M. Smith. 2006. Crop evapotranspiration- Guidelines for computing crop water requirements-FAO. Irrigation and drainage. Water Resources, Development and Management Service, Rome, Italy, 1998, paper 56. Pp 323.

Allison, J. D., D. S. Brown, and K. J. Novo-Gradac. 1991. MINTEQA2/PRODEFA2, a geochemical assessment model for environmental systems: version 3.0 user's manual. Environmental Research Laboratory, Office of Research and Development, US Environmental Protection Agency.

Amiro, B. D., and L. L. Ewing. 1992. Physiological conditions and uptake of inorganic carbon-14 by plant roots. Environmental and Experimental Botany 32(3):203-211.

Appelo, C. A. J., and D. Postma. 2004. Geochemistry, groundwater and pollution. CRC press.

Arnold, G. 1992. Soil acidification as caused by the nitrogen uptake pattern of Scots pine (Pinus sylvestris). Plant and Soil 142(1):41-51.

Baldi, G., and J. Paruelo. 2008. Land-Use and Land Cover Dynamics in South American Temperate Grasslands. Ecology and Society 13(2):6. https:/ / doi.org/10.5751/ES-02481-130206.

Battaglia, M., and P. Sands. 1997. Modelling site productivity of Eucalyptus globulus in response to climatic and site factors. Functional Plant Biology 24(6):831-850. https://doi.org/10.1071/PP97065

BDH. 2019. Base de Datos Hidrológicos. URL: http://www.bdh.acumar.gov.ar/bdh3/index_contenido.php (último acceso octubre 2019).

Bea, S. A., H. Wainwright, N. Spycher, B. Faybishenko, S. S. Hubbard, and M. E. Denham. 2013. Identifying key controls on the behavior of an acidic-U (VI) plume in the Savannah River Site using reactive transport modeling. Journal of Contaminant Hydrology 151:34-54.

Bea, S. A., S. A. Wilson, K. U. Mayer, G. M. Dipple, I. M. Power, and P. Gamazo. 2012. Reactive Transport Modeling of Natural Carbon Sequestration in Ultramafic Mine Tailings. Vadose Zone Journal 11(2). https:/ / doi.org/10.2136/ vzj2011.0053.

Bea, S. A., U. K. Mayer, and K. T. B. MacQuarrie. 2016. Reactive transport and thermolhydrolmechanical coupling in deep sedimentary basins affected by glaciation cycles: model development, verification, and illustrative example. Geofluids 16(2):279-300.

Berthrong, S. T., E. G. Jobbágy, and R. B. Jackson. 2009. A global metalanalysis of soil exchangeable cations, pH, carbon, and nitrogen with afforestation. Ecological Applications 19(8):2228-2241.

Besteiro, S. 2014. Evaluación de la influencia hidrológica de forestaciones en la llanura pampeana. (tesis de doctorado). URL: sedici.unlp.edu.ar/handle/10915/33806. La Plata, Buenos Aires: Universidad Nacional de La Plata.

Bowman, W. D., C. C. Cleveland, L. Halada, J. Hreško, and J. S. Baron. 2008. Negative impact of nitrogen deposition on soil buffering capacity. Nature Geoscience 1(11):767.

Burkart, S. E., M. F. Garbulsky, C. M. Ghersa, J. P. Guerschman, R. J. C. León, et al. 2005. Las comunidades potenciales del pastizal pampeano bonaerense. La Heterogeneidad de La Vegetación de Los Agroecosistemas. Un Homenaje a Rolando León. Ed. Facultad de Agronomía. Buenos Aires, Argentina. Pp. 379-400.

Cabrera, A. L. 1976. Regiones fitogeográficas argentinas. Enciclopedia Argentina de Agricultura y Jardinería (2da. ed.). Tomo II, Fase 1 ACME, Buenos Aires. Pp. 85.

Calder, I. R. 1998. Water use by forests, limits and controls. Tree Physiology 18(8-9):625-631. https://doi.org/10.1093/ treephys/18.8-9.625

Carol, E., E. Kruse, and J. Pousa. 2008. Environmental hydrogeology of the southern sector of the Samborombón Bay wetland, Argentina. Environmental Geology 54(1):95-102.

Carretero, S. C., C. Dapeña, and E. E. Kruse. 2013. Hydrogeochemical and isotopic characterization of groundwater in a sand-dune phreatic aquifer on the northeastern coast of the province of Buenos Aires, Argentina. Isotopes in Environmental and Health Studies 49(3):399-419.

Dakora, F. D., and D. A. Phillips. 2002. Root exudates as mediators of mineral acquisition in low-nutrient environments. In Food Security in Nutrient-Stressed Environments: Exploiting Plants' Genetic Capabilities. Springer, Dordrecht. Pp. 201-213.

Engel, V., E. G. Jobbágy, M. Stieglitz, M. Williams, and R. B. Jackson. 2005. Hydrological consequences of Eucalyptus afforestation in the Argentine Pampas. Water Resources Research 41(10):1-14. https://doi.org/10.1029/ 2004WR003761.

Epron, D., Y. Nouvellon, O. Roupsard, W. Mouvondy, A. Mabiala, et al. 2004. Spatial and temporal variations of soil respiration in a Eucalyptus plantation in Congo. Forest Ecology and Management 202(1-3):149-160. https://doi.org/ 10.1016/j.foreco.2004.07.019.

Fuchs, R., M. Herold, P. H. Verburg, J. G. Clevers, and J. Eberle. 2015. Gross changes in reconstructions of historic land cover/use for Europe between 1900 and 2010. Global Change Biology 21(1):299-313. 
Gérard, F., C. Blitz-Frayret, P. Hinsinger, and L. Pagès. 2017. Modelling the interactions between root system architecture, root functions and reactive transport processes in soil. Plant and Soil 413(1-2):161-180. https://doi.org/10.1007/ s11104-016-3092-x.

Heuperman, A. 1999. Hydraulic gradient reversal by trees in shallow water table areas and repercussions for the sustainability of tree-growing systems. Agricultural Water Management 39(2-3):153-167. https://doi.org/10.1016/ S0378-3774(98)00076-6.

Hinsinger, P., G. R. Gobran, P. J. Gregory, and W. W. Wenzel. 2005. Rhizosphere geometry and heterogeneity arising from root mediated physical and chemical processes. New Phytologist 168:293-303. https://doi.org/10.1111/j.14698137.2005.01512.x.

Hong, S., P. Gan, and A. Chen. 2019. Environmental controls on soil pH in planted forest and its response to nitrogen deposition. Environmental Research 172:159-165. https:/ / doi.org/S0013935119300933.

Hong, S., S. Piao, A. Chen, Y. Liu, L. Liu, et al. 2018. Afforestation neutralizes soil pH. Nature Communications 9(1): 520 .

Hopmans, J. W., and K. L. Bristow. 2002. Current capabilities and future needs of root water and nutrient uptake modeling. Pp. 103-183 in Advances in Agronomy. Vol. 77. Academic Press.

Ickowitz, A., B. Powell, D. Rowland, A. Jones, and T. Sunderland. 2019. Agricultural intensification, dietary diversity, and markets in the global food security narrative. Global Food Security 20:9-16. doi:10.1016/j.gfs.2018.11.002.

INTA. 2011. Cartas de Suelos de la República Argentina. Hoja 3757-02 - DOLORES. CIRN, INTA, Buenos Aires. URL: anterior.inta.gov.ar/suelos/cartas/3757/Dolores/index.htm.

Jackson, R. B., J. Canadell, J. R. Ehleringer, H. A. Mooney, O. E. Sala, and E. D. Schulze. 1996. A global analysis of root distributions for terrestrial biomes. Oecologia 108(3):389-411. https://doi.org/10.1007/BF00333714.

Jackson, R. B., S. R. Carpenter, C. N. Dahm, D. M. McKnight, R. J. Naiman, S. L. Postel, and S. W. Running. 2001. Water in a changing world. Ecological Applications 11(4):1027-1045. https://doi.org/10.1890/1051-0761(2001)011[1027. WIACW]2.0.CO;2

Jobbágy, E. G., and Jackson, R. B. 2003. Patterns and Mechanisms of Soil Acidification in the Conversion of Grasslands to Forests 64(2):205-229.

Jobbágy, E. G., and R. B. Jackson. 2001. The distribution of soil nutriments with depth: Global patterns of the imprint of plants. Biogeochemistry 53:51-77.

Jobbágy, E. G., and R. B. Jackson. 2004. Groundwater use and salinization with grassland afforestation. Global Change Biology 10(8):1299-1312. https:// doi.org/10.1111/j.1365-2486.2004.00806.x.

Jobbágy, E. G., and R. B. Jackson. 2007. Groundwater and soil chemical changes under phreatophytic tree plantations. Journal of Geophysical Research: Biogeosciences 112(2):1-15. https://doi.org/10.1029/2006]G000246.

Jobbágy, E. G., G. Piñeiro, M. D. Nosetto, and J. M. Paruelo. 2006. Las forestaciones rioplatenses y el agua. Ciencia Hoy 16(95):12-21.

Jobbágy, E. G., T. Tóth, M. D. Nosetto, and S. Earman. 2017. On the Fundamental Causes of High Environmental Alkalinity $(\mathrm{pH} \geq 9)$ : An Assessment of Its Drivers and Global Distribution. Land Degradation and Development 28(7):1973-1981. https://doi.org/10.1002/ldr.2718.

Kunito, T., I. Isomura, H. Sumi, H. D. Park, H. Toda, et al. 2016. Aluminum and acidity suppress microbial activity and biomass in acidic forest soils. Soil Biology and Biochemistry 97:23-30.

Lal, R. 2003. Offsetting global CO2 emissions by restoration of degraded soils and intensification of world agriculture and forestry. Land Degradation and Development 14(3):309-322. https://doi.org/10.1002/ldr.562.

Mayer, K. U., E. O. Frind, and D. W. Blowes. 2002. Multicomponent reactive transport modeling in variably saturated porous media using a generalized formulation for kinetically controlled reactions. Water Resources Research 38(9): 1174. https://doi.org/10.1029/2001WR000862.

Mayer, K., R. Amos, S. Molins, and F. Gerard. 2012. Reactive transport modeling in variably saturated media with min3p: Basic model formulation and model enhancements. Groundwater Reactive Transport Models. Pp. 186-211. https://doi.org/10.2174/978160805306311201010186.

Milione G. M., C. R. Mujica, S. A. Bea, D. Domínguez Daguer, and J. E. Gyenge. 2018. Forestación en pastizales: el rol de las especies y el manejo forestal sobre el proceso de salinización secundaria de suelos. Revista de investigaciones agropecuarias (RIA). ISSN 1669-2314. En prensa.

Miller, R., B. Wesley, and P. Norman. 1988. Specific Conductance: Theoretical Considerations and Application to Analytical Quality Control. United States Geological Survey Water-Supply 2311:3-6.

Modernel, P., W. A. Rossing, M. Corbeels, S. Dogliotti, V. Picasso, and P. Tittonell. 2016. Land use change and ecosystem service provision in Pampas and Campos grasslands of southern South America. Environmental Research Letters 11(11):113002.

Nosetto, M. D., E. G. Jobbágy, A. B. Brizuela, and R. B. Jackson. 2012. The hydrologic consequences of land cover change in central Argentina. Agriculture, Ecosystems and Environment 154:2-11. https://doi.org/10.1016/ j.agee.2011.01.008.

Nosetto, M. D., E. G. Jobbágy, T. Tóth, and C. M. Di Bella. 2007. The effects of tree establishment on water and salt dynamics in naturally salt-affected grasslands. Oecologia 152(4):695-705. https:/ / doi.org/10.1007/s00442-007-06942 .

Nosetto, M. D., E. G. Jobbágy, T. Tóth, and R. B. Jackson. 2008. Regional patterns and controls of ecosystem salinization with grassland afforestation along a rainfall gradient. Global Biogeochemical Cycles 22(2):1-12. https://doi.org/ 
10.1029/2007GB003000.

Nowack, B., K. U. Mayer, S. E. Oswald, W. Van Beinum, C. A. J. Appelo, et al. 2006. Verification and intercomparison of reactive transport codes to describe root-uptake. Plant and Soil 285(1-2):305-321. https://doi.org/10.1007/s11104006-9017-3.

Nye, P. H. 1981. Changes of $\mathrm{pH}$ across the rhizosphere induced by roots. Plant and Soil 61(1-2):7-26. https://doi.org/ $10.1007 /$ BF02277359.

O'Connell, A. M. 1987. Litter decomposition, soil respiration and soil chemical and biochemical properties at three contrasting sites in karri (Eucalyptus diversicolor F. Muell.) forests of south】western Australia. Australian Journal of Ecology 12(1):31-40. https:/ / doi.org/10.1111/j.1442-9993.1987.tb00925.x.

Parkhurst, D. L., and C. A. J. Appelo. 1999. User's guide to PHREEQC (version 2)-a computer program for speciation, batch-reaction, one-dimensional transport, and inverse geochemical calculations. Water-Resources Investigations Report 99(4259):312.

Raich, J. W., and A. Tufekcioglu. 2000. Vegetation and Soil Respiration: Correlations and Controls. Biogeochemistry 48(1):71-90.

Ramankutty, N., Z. Mehrabi, K. Waha, L. Jarvis, C. Kremen, M. Herrero, and L. H. Rieseberg. 2018. Trends in global agricultural land use: implications for environmental health and food security. Annual Review of Plant Biology 69: 789-815.

Rhoades, J. D., N. A. Manteghi, P. J. Shouse, and W. J. Alves. 1989. Soil Electrical Conductivity and Soil Salinity: New Formulations and Calibrations. Soil Science Society of America Journal 53(2):433. https://doi.org/10.2136/ sssaj1989.03615995005300020020x.

Richter, D. D. 1986. Sources of Acidity in Some Forested Udults 1. Soil Science Society of America Journal 50(6):15841589.

Saviozzi, A., R. Levi-Minzi, R. Cardelli, and R. Riffaldi. 2001. A comparison of soil quality in adjacent cultivated, forest and native grassland soils. Plant and Soil 233(2):251-259.

Schaap, M. G., F. J. Leij, and M. T. Van Genuchten. 2001. Rosetta: A computer program for estimating soil hydraulic parameters with hierarchical pedotransfer functions. Journal of Hydrology 251(3-4):163-176. https:/ / doi.org/10.1016/ S0022-1694(01)00466-8.

Šimůnek, J., and J. W. Hopmans. 2009. Modeling compensated root water and nutrient uptake. Ecological Modelling 220(4):505-521

Soriano, A. 1991. Río de la Plata grasslands. Pp. 367-405 in Natural Grasslands: Introduction and Western Hemisphere. R. T. Coupland (ed.). Elsevier, New York.

Steefel, C. I., C. A. J. Appelo, B. Arora, D. Jacques, T. Kalbacher, et al. 2014. Reactive transport codes for subsurface environmental simulation. Computational Geosciences 19(3):445-478. doi:10.1007/s10596-014-9443-X.

Sumner, M. E. 1993. Sodic soils-New perspectives. Soil Research 31(6):683-750.

Teakle, N. L., and S. D. Tyerman. 2010. Mechanisms of Cl- transport contributing to salt tolerance. Plant, Cell and Environment 33(4):566-589. https://doi.org/10.1111/j.1365-3040.2009.02060.x.

Teruggi, M. E. 1957. The Nature and Origin of Argentine Loess. SEPM Journal of Sedimentary Research 27(3):322-332. https://doi.org/10.1306/74D706DC-2B21-11D7-8648000102C1865D

Tricart, J. L. 1973. Geomorfología de la Pampa Deprimida: Base Para los Estudios Edafológicos y Agronómicos, Colección Cient. Vol. 12. Inst. Nac. Tecnol. Agropecuaria, Buenos Aires. Pp. 202.

van Breemen, N., and P. Buurman. 2002. Soil formation. Springer Science and Business Media.

van Breemen, N., J. Mulder, and C. T. Driscoll. 1983. Acidification and alkalinization of soils. Plant and Soil 75(3):283308. https://doi.org/10.1007/BF02369968.

van Genuchten, M. T. 1980. Closed-Form Equation for Predicting the Hydraulic Conductivity of Unsaturated Soils. Soil Science Society of America Journal 44(5):892-898.

Varni, M. R., and E. J. Usunoff. 1999. Simulation of regional-scale groundwater flow in the Azul River basin, Buenos Aires Province, Argentina. Hydrogeology Journal 7(2):180-187. https://doi.org/10.1007/s100400050190.

Viglizzo, E. F., F. Lértora, A. J. Pordomingo, J. N. Bernardos, Z. E. Roberto, and H. Del Valle. 2001. Ecological lessons and applications from one century of low external-input farming in the pampas of Argentina. Agriculture, Ecosystems and Environment. https://doi.org/10.1016/S0167-8809(00)00155-9

Xie, M., K. Mayer, F. Claret, P. Alt-Epping, D. Jacques, C. Steefel, C. Chiaberge, and J. Simunek. 2014. Implementation and evaluation of permeability-porosity and tortuosity-porosity relationships linked to mineral dissolution-precipitation. Computational Geosciences 19:655-71.

Zabala, M. E., S. Martínez, M. Manzano, and L. Vives. 2016. Groundwater chemical baseline values to assess the Recovery Plan in the Matanza-Riachuelo River basin, Argentina. Science of the Total Environment 541:1516-1530. https://doi.org/10.1016/j.scitotenv.2015.10.006.

Zárate, M. A. 2003. Loess of southern South America. Quaternary Science Reviews 22(18-19):1987-2006. https:/ doi.org/10.1016/S0277-3791(03)00165-3 